\title{
A Contribution to the Fauna of Asilidae (Diptera, Brachycera) in Fars Province, Southern Iran
}

\author{
N. SAGHAEI* \\ Department of Entomology, Marvdasht Branch, Islamic Azad University, Marvdasht, Iran
}

(Received: 28 January 2015; accepted: 20 March 2015)

\begin{abstract}
A survey was conducted to study the species of Asilidae (Diptera, Brachycera) in Fars province, southern Iran during year 2012. A total of 12 species belonging to 12 genera and 6 subfamilies Apocleinae, Asilinae, Dasypogoninae, Leptogastrinae, Ommatiinae and Stenopogoninae were collected and identified. Among them, Tolmerus paganus Becker, 1923 is recorded for the first time in Iran. Additionally one subfamily, two genera and four species are recorded for the first time from Fars province. Distributional data for each species are given. An updated list of all known species of Asilidae from Fars province is also included.
\end{abstract}

Keywords: Diptera, distribution, Fars province, fauna, Iran, robber flies.

Asilidae is a large, diverse, cosmopolitan family of flies commonly known as robber flies that consists of over 7000 species and subspecies in 530 genera, but with many further species yet to be described (Geller-Grimm et al., 2015). The name "robber flies" reflects their aggressive predatory habits; they feed mainly on other arthropods, which may help to maintain a balance between insect populations in various ecosystems (Londt, 1993, 1994, 1995, 1996, 2006; Dennis and Lavigne, 2007). The majority of species of Asilidae frequent in the warm temperate and tropical areas of the world (Theodor, 1980; Wood, 1981).

In recent years some faunistical studies have been conducted on Iranian Asilidae and currently the number of species of this family recorded in Iran has grown (Tomasovic, 2002; Lehr et al., 2007; Saghaei et al., 2008, 2009, 2010). Hayat et al. (2008) provided a checklist of the Iranian species, including of 156 species and subspecies. Later, Saghaei et al. (2009) have added some species records from Iran and have provided a list of 232 asilid species and subspecies from Iran. Also, Tomasovic and Saghaei (2009) discribed three new species and one new subspecies from the country. However, the Asilidae fauna of Iran is still not fully studied.

Fars province (coordinates $27^{\circ}-31^{\circ} \mathrm{N}, 50^{\circ}-55^{\circ} \mathrm{E}$ ), with an area of about $1,333,000 \mathrm{~km}^{2}$, is located in the southern part of Iran (Fig. 1). Fars consists of three completely different regions. (1) The mountain area north and northwest, with moderate cold

*E-mail: nazila_saghaei@yahoo.com 


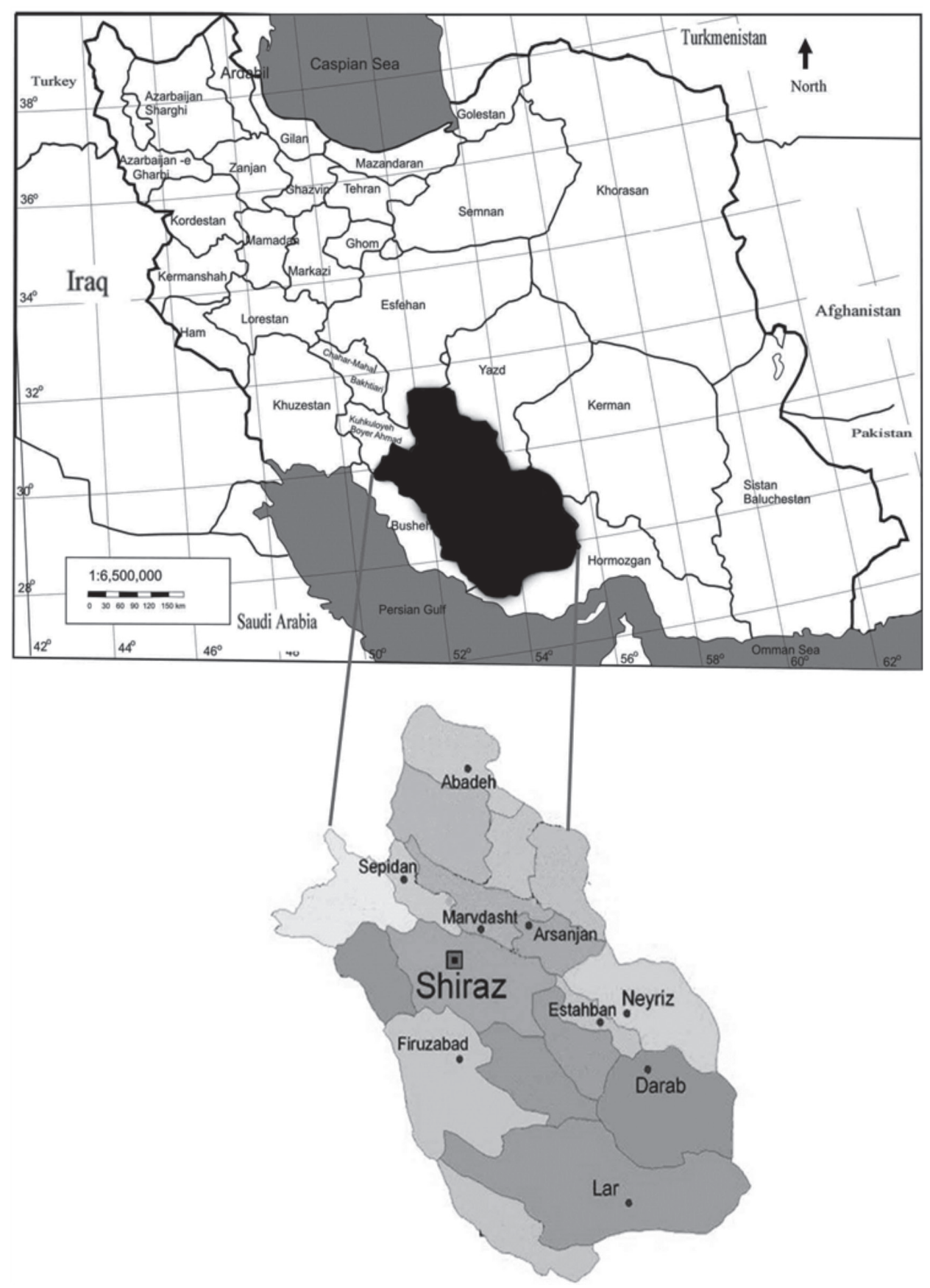

Fig. 1. Map of Iran and Fars province. Upper panel: Location of Fars province (black) in Iran; Lower panel: Location of the sampling localities in Fars province 
winters and mild summers, (2) the central region, with relatively rainy mild winters, and hot dry summers, (3) the south and southeast, with moderate winters and very hot summers. This province with having such climate and geographic diversity is a very interesting area with a richly varied fauna and flora of native species.

The present article continues a series of publications on the asilid fly fauna from Fars province (Saghaei et al., 2008, 2009, 2010; Tomasovic and Saghaei, 2009). Here, I provide some additional data on Asilidae in South-West Iran, add one species to the published records, and provide an updated list of all known species of Asilidae from Fars province.

\section{Materials and Methods}

The specimens were collected using a standard Malaise trap from different places (i.e. Marvdasht, Shiraz, Darab, Abadeh, Neyriz, Arsanjan, Estahban, Sepidan and Lar) in Fars province, Iran. The collected specimens were pinned, labeled, and put into collection boxes, subsequently. The voucher specimens are deposited in the Department of Entomology, College of Agriculture, Marvdasht Branch, Islamic Azad University, Marvdasht, Iran. Identifications were made using published keys of Engel (1930), Oldroyd (1958), Abbassian-Lintzen (1964), Tsacas (1968), Theodor (1980) and Lehr (1988). The taxonomic arrangement and distributional data for regions outside Iran used in this paper were adapted from Lehr (1988), Bosák and Hradský (2001), Hayat et al. (2008) and Geller-Grimm et al. (2015).

\section{Results}

The asilid species collected and identified during this study are listed as follows:

\section{Subfamily Apocleinae Papavero, 1973 Genus Apoclea Macquart 1838}

\section{Apoclea femoralis (Wiedemann, 1828)}

Distribution in Iran: Iran (Engel, 1930; Theodor 1980), Kerman, Khorasan (Becker and Stein, 1913), Baluchestan (Becker and Stein, 1913; Oldroyd 1958), Khuzestan, Mazandaran (Ghahari et al., 2007a), Golestan, Guilan (Hayat et al., 2008); Fars (Saghaei et al., 2008, 2009, 2010; Tomasovic and Saghaei, 2009).

Distribution outside Iran: Afghanistan, Azerbaijan, Greece, Iraq, Israel, Russia, Turkey.

\section{Subfamily Asilinae Latreille, 1802 \\ Genus Aneomochtherus Lehr, 1996}

\section{Aneomochtherus perplexus (Becker, 1923)}

Material examined: 3 우: Marvdasht, $2^{\circ} 52^{\prime} \mathrm{N}, 5^{\circ} 48^{\prime} \mathrm{E}, 11 . \mathrm{V} .2012 ; 4$ 우: Arsanjan, $29^{\circ} 29^{\prime} \mathrm{N}, 5^{\circ} 15^{\prime} \mathrm{E}, 22$. VI.2012; 1O : Estahban, $29^{\circ} 08^{\prime} \mathrm{N}, 5^{\circ} 03{ }^{\prime} \mathrm{E}, 10 . I X .2012$. 
Distribution in Iran: Iran (Tsacas, 1968), Khuzestan (Oldroyd, 1958), Khuzestan, Mazandaran (Ghahari et al., 2007a), Guilan (Hayat et al., 2008), Fars (Tomasovic and Saghaei, 2009), Semnan (Sakenin et al., 2010).

Distribution outside Iran: Armenia, Azerbaijan, Russia, Turkey, Ukraine.

\section{Genus Antiphrisson Loew, 1849}

\section{Antiphrisson adpressus (Loew, 1849)}

Material examined: 1o: Marvdasht, $29^{\circ} 52^{\prime} \mathrm{N}, 5^{\circ} 48^{\prime} \mathrm{E}, 18$. V.2012; 1o: Estahban, $29^{\circ} 08^{\prime} \mathrm{N}, 5^{\circ} 03^{\prime} \mathrm{E}, 10 . \mathrm{IX} .2012$.

Distribution in Iran: Khuzestan (Oldroyd, 1958), Fars (Saghaei et al., 2008, 2009, 2010; Tomasovic and Saghaei, 2009).

Distribution outside Iran: Azerbaijan, Egypt, Israel, Kazakhstan, Russia, Tunisia, Turkey.

\section{Genus Satanas Jacobson, 1908}

\section{Satanas gigas (Eversmann, 1855)}

Material examined: 1o: $\mathrm{Lar}^{\circ} 7^{\circ} 32^{\prime} \mathrm{N}, 5^{\circ} 22^{\prime} \mathrm{E}$, 5.VI.2012.

Distribution in Iran: Baluchestan, Kerman, Khorasan, Sistan (Becker and Stein, 1913), Iran (Engel, 1930), Baluchestan (Oldroyd, 1958), Mazandaran (Ghahari et al., 2007a), Semnan (Hayat et al., 2008; Sakenin et al., 2010), Fars (Saghaei et al., 2008, 2009, 2010; Tomasovic and Saghaei, 2009).

Distribution outside Iran: Algeria, Azerbaijan, China, Egypt, Greece, Israel, Kazakhstan, Libya, Mongolia, Romania, Russia, Turkey.

\section{Genus Tolmerus Loew, 1849}

\section{Tolmerus paganus Becker, 1923}

Material examined: 1Q: Marvdasht, $29^{\circ} 52^{\prime} \mathrm{N}, 52^{\circ} 48^{\prime} \mathrm{E}, 29 . \mathrm{V} .2012 ; 1$ \%: Shiraz,

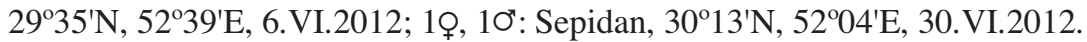

Distribution outside Iran: Russia South. This is a new record for the fauna of Fars province and Iran.

\section{Subfamily Dasypogoninae Macquart, 1838 \\ Tribe Dasypogonini Macquart, 1838 \\ Genus Dasypogon Meigen, 1803}

\section{Dasypogon magisi Tomasovic, 1999}

Material examined: 2ǫ̊: Lar, $27^{\circ} 32^{\prime} \mathrm{N}, 5^{\circ} 22^{\prime} \mathrm{E}, 5 . \mathrm{VI} .2012 ; 1$; : Abadeh, $31^{\circ} 09^{\prime} \mathrm{N}$, 52³7'E, 14.VII. 2012.

Distribution in Iran: Fars (Tomasovic, 1999; Saghaei et al., 2008, 2009, 2010; Tomasovic and Saghaei, 2009).

Distribution outside Iran: Iraq. 


\section{Subfamily Leptogastrinae Schiner, 1862 \\ Genus Leptogaster Meigen, 1803}

\section{Leptogaster nartshukae Lehr, 1961}

Material examined: 1Q: Darab, $28^{\circ} 40^{\prime} \mathrm{N}, 54^{\circ} 50^{\prime} \mathrm{E}, 19 . \mathrm{V} .2012$.

Distribution in Iran: Iran (Lehr, 1988). This is a new record for the fauna of Fars province.

Distribution outside Iran: Kazakhstan, Mongolia, Russia (West and East Siberia).

\section{Subfamily Ommatiinae Hardy, 1927 \\ Genus Ommatius Wiedemann, 1821}

\section{Ommatius tenellus Wulp, 1889}

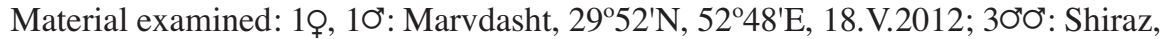

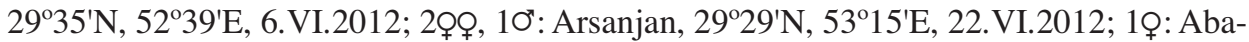
deh, $31^{\circ} 09^{\prime} \mathrm{N}, 52^{\circ} 37^{\prime} \mathrm{E}, 14$. VII.2012; 30'0': Estahban, $29^{\circ} 08^{\prime} \mathrm{N}, 54^{\circ} 03^{\prime} \mathrm{E}, 10$. IX.2012; $30^{\circ} 0^{\prime \prime}$ : Neyriz, $29^{\circ} 16^{\prime} \mathrm{N}, 5^{\circ} 19^{\prime} \mathrm{E}, 12 . \mathrm{IX} .2012$.

Distribution in Iran: Baluchestan (Oldroyd, 1958). This is a new record for the fauna of Fars province.

Distribution outside Iran: Algeria, Azerbaijan, Chad, China, Egypt, Ethiopia, India, Israel, Niger, Senegal, Sudan, Yemen.

\section{Subfamily Stenopogoninae Hull, 1962 \\ Tribe Stichopogonini Hardy, 1930 \\ Genus Stichopogon Loew, 1847}

\section{Stichopogon chrysostoma Schiner, 1867}

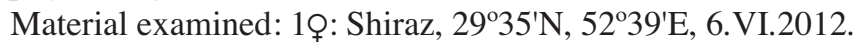

Distribution in Iran: Khorasan (Becker and Stein, 1913), Tehran (Abbassian-Lintzen, 1964). This is a new record for the fauna of Fars province.

Distribution outside Iran: Cyprus, Egypt, Israel, Kazakhstan, Libya, Mongolia, former Soviet Middle Asia, Turkey.

\section{Tribe Stenopogonini Hardy, 1930 \\ Genus Galactopogon Engel, 1929}

\section{Galactopogon hispidus Engel, 1929}

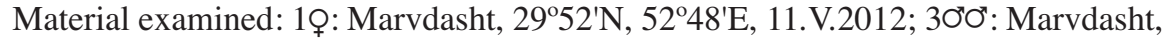

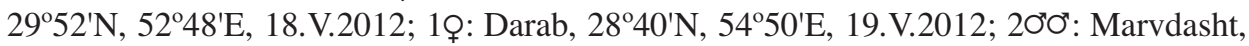

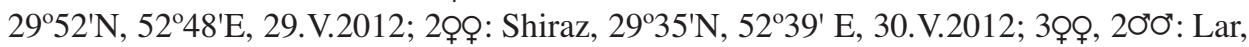

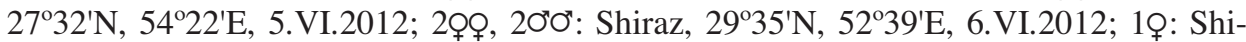

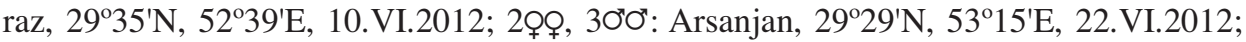

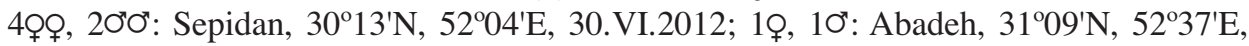


14.VII.2012; 2Q̊, 10': Estahban, $29^{\circ} 08^{\prime} \mathrm{N}, 5^{\circ} 03^{\prime} \mathrm{E}, 10 . \mathrm{IX} .2012 ; 2$ \%ᄋ, Neyriz, $29^{\circ} 16^{\prime} \mathrm{N}$, 54ำ'E, 12.IX.2012.

Distribution in Iran: Baluchestan, Khuzestan (Oldroyd, 1958), Khuzestan, SE Iran (Abbassian-Lintzen, 1964), Fars (Abbassian-Lintzen, 1964; Saghaei et al., 2008, 2009, 2010).

Distribution outside Iran: Algeria, Jordan, Israel.

\section{Genus Stenopogon Loew, 1847}

\section{Stenopogon sciron (Loew, 1873)}

Material examined: 10: Marvdasht, 29 $52^{\prime} \mathrm{N}, 5^{\circ} 48^{\prime} \mathrm{E}, 11 . \mathrm{V} .2012 ; 10^{\star}$ : Abadeh, $31^{\circ} 09^{\prime} \mathrm{N}, 52^{\circ} 37^{\prime} \mathrm{E}, 14$. VII.2012;

Distribution in Iran: Kerman (Abbassian-Lintzen, 1964), Iran (Engel, 1930; Theodor, 1980), Fars (Saghaei et al., 2008, 2009, 2010; Tomasovic and Saghaei, 2009).

Distribution outside Iran: Afghanistan, Azerbaijan, China, Egypt, Turkey.

\section{Table 1}

List of the Asilidae known from Fars province south of Iran

\begin{tabular}{|c|c|c|}
\hline No. & Subfamily, Tribe, Genus and Species & References \\
\hline & \multicolumn{2}{|c|}{ Subfamily Apocleinae Papavero, 1973} \\
\hline & \multicolumn{2}{|c|}{ Genus Apoclea Macquart 1838} \\
\hline \multirow[t]{2}{*}{1} & Apoclea femoralis (Wiedemann, 1828) & $\begin{array}{c}\text { Saghaei et al., 2008, 2009, 2010; } \\
\text { Tomasovic and Saghaei, 2009; } \\
\text { Present study }\end{array}$ \\
\hline & \multicolumn{2}{|c|}{ Genus Philodicus Loew, 1847} \\
\hline 2 & Philodicus ponticus (Bigot, 1880) & $\begin{array}{c}\text { Saghaei et al., 2008, 2009, 2010; } \\
\text { Tomasovic and Saghaei, 2009; } \\
\text { Present study }\end{array}$ \\
\hline \multirow[t]{2}{*}{3} & Philodicus spectabilis Loew, 1871 & $\begin{array}{l}\text { Saghaei et al., 2008, 2009, 2010; } \\
\text { Tomasovic and Saghaei, } 2009\end{array}$ \\
\hline & \multicolumn{2}{|c|}{ Genus Promachus Loew, 1848} \\
\hline 4 & Promachus canus (Wiedemann, 1818) & Saghaei et al., 2008, 2009, 2010 \\
\hline 5 & Promachus leoninus Loew, 1848 & $\begin{array}{l}\text { Saghaei et al., 2008, 2009; } \\
\text { Tomasovic and Saghaei, } 2009\end{array}$ \\
\hline \multirow[t]{3}{*}{6} & Promachus maculatus (Fabricius, 1775) & Sakenin et al., 2008 \\
\hline & \multicolumn{2}{|c|}{ Subfamily Asilinae Latreille, 1802} \\
\hline & \multicolumn{2}{|c|}{ Genus Aneomochtherus Lehr, 1996} \\
\hline 7 & Aneomochtherus macropygus Tsacas, 1968 & Saghaei et al., 2010 \\
\hline
\end{tabular}


Table 1 (cont.)

\begin{tabular}{rlc}
\hline No. & \multicolumn{1}{c}{ Subfamily, Tribe, Genus and Species } & References \\
\hline 8 & Aneomochtherus mundus (Loew, 1849) & Saghaei et al., 2010 \\
9 & Aneomochtherus ochriventris (Loew, 1854) & Saghaei et al., 2008; Tomasovic and Saghaei, 2009 \\
10 & A. ochriventris caucasicus Tsacas, 1968 & Saghaei et al., 2010 \\
11 & $\begin{array}{l}\text { A. ochriventris persicus Tomasovic and } \\
\text { Saghaei, 2009 }\end{array}$ & Tomasovic and Saghaei, 2009 \\
12 & Aneomochtherus perplexus (Becker, 1923) & Tomasovic and Saghaei, 2009; \\
& & Present study
\end{tabular}

\section{Genus Antipalus Loew, 1849}

13 Antipalus truncatus (Loew, 1849)

Lehr et al., 2007

\section{Genus Antiphrisson Loew, 1849}

14 Antiphrisson adpressus (Loew, 1849)

Saghaei et al., 2008, 2009, 2010; Tomasovic and Saghaei, 2009; Present study

15 Antiphrisson trifarius (Loew, 1849) Saghaei et al., 2008, 2009, 2010

\section{Genus Cerdistus Loew, 1849}

16 Cerdistus zelleri Schiner, 1862

Samin et al., 2011a

Genus Didysmachus Lehr, 1996

17 Didysmachus picipes (Meigen, 1820)

Lehr et al., 2007

Genus Dysmachus Loew, 1860

18 Dysmachus cephalenus Loew, 1871

Lehr et al., 2007

19 Dysmachus fuscipennis (Meigen, 1820)

Lehr et al., 2007

20 Dysmachus praemorsus (Loew, 1854)

Lehr et al., 2007

Genus Echthistus Loew, 1849

21 Echthistus rufinervis (Meigen, 1820)

Lehr et al., 2007

\section{Genus Engelepogon Lehr, 1992}

22 Engelepogon idiorrhytmicus (Janssen, 1960)

Saghaei et al., 2008, 2009, 2010

\section{Genus Erax Scolopi, 1763}

23 Erax grootaerti Tomasovic, 2002

24 Erax tenuicornis (Loew, 1848)

25 Eutolmus mordax (Loew, 1848)
Tomasovic 2002; Tomasovic and Saghaei, 2009

Samin et al., 2011a

Genus Eutolmus Loew, 1848

Lehr et al., 2007

Genus Machimus Loew, 1849

26 Machimus annulipes (Brulle, 1832)

Saghaei et al., 2010

27 Machimus aradensis Theodor, 1980

Saghaei et al., 2008, 2009, 2010

Sakenin et al., 2008

28 Machimus caliginosus (Meigen, 1820)

Saghaei et al., 2008, 2009, 2010 
Table 1 (cont.)

\begin{tabular}{llc}
\hline No. & \multicolumn{1}{c}{ Subfamily, Tribe, Genus and Species } & References \\
\hline 30 & Machimus rudis Becker, 1923 & Lehr et al., 2007 \\
31 & Machimus rusticus (Meigen, 1820) & Saghaei et al., 2008, 2009, 2010; \\
& & Tomasovic and Saghaei, 2009 \\
& & Genus Paramochtherus Theodor, 1980
\end{tabular}

Genus Philonicus Loew, 1849

33 Philonicus albiceps (Meigen, 1820)

Saghaei et al., 2010;

Tomasovic and Saghaei, 2009

Genus Satanas Jacobson, 1908

34 Satanas gigas (Eversmann, 1855)

Saghaei et al., 2008, 2009, 2010;

Tomasovic and Saghaei, 2009; Present study

Genus Tolmerus Loew, 1849

35 Tolmerus atricapillus (Fallen, 1814)

Sakenin et al., 2008

36 Tolmerus paganus Becker, 1923

Present study

Genus Trichomachimus Engel, 1934

37 Trichomachimus pubescens Moucha and

Sakenin et al., 2008

Hradský, 1964

Subfamily Dasypogoninae Macquart, 1838

Tribe Dasypogonini Macquart, 1838

Genus Dasypogon Meigen, 1803

38 Dasypogon diadema (Fabricius, 1781)

Abbassian-Lintzen, 1964

39 Dasypogon irinelae Weinberg, 1986

Saghaei et al., 2010

40 Dasypogon magisi Tomasovic, 1999

Tomasovic, 1999; Saghaei et al., 2008, 2009, 2010; Tomasovic and Saghaei, 2009; Present study

Genus Saropogon Loew, 1847

41 Saropogon albicans Janssens, 1961

Sakenin et al., 2008

42 Saropogon distinctus Becker, 1906

Saghaei et al., 2008, 2009, 2010

43 Saropogon leucocephalus (Meigen, 1820)

Sakenin et al., 2008

Subfamily Laphriinae Macquart, 1838

Tribe Laphriini Macquart, 1838

Genus Choerades Walker, 1851

44 Choerades fuliginosa (Panzer, 1798)

Samin et al., 2011b

45 Choerades fulva (Meigen, 1804)

Samin et al., 2011a

\section{Genus Laphria Meigen, 1803}

46 Laphria dizonias Loew, 1847

Abbassian-Lintzen, 1964; Saghaei et al., 2008, 2009, 2010; Tomasovic and Saghaei, 2009 
Table 1 (cont.)

\begin{tabular}{|c|c|c|}
\hline No. & Subfamily, Tribe, Genus and Species & References \\
\hline \multicolumn{3}{|c|}{ Tribe Andrenosomini Hull, 1962} \\
\hline \multicolumn{3}{|c|}{ Genus Andrenosoma Ronndani, 1856} \\
\hline 47 & $\begin{array}{l}\text { Andrenosoma farsicola Tomasovic and } \\
\text { Saghaei, } 2009\end{array}$ & Tomasovic and Saghaei, 2009 \\
\hline \multicolumn{3}{|c|}{ Tribe Ctenotini Hull, 1962} \\
\hline \multicolumn{3}{|c|}{ Genus Stiphrolamyra Engel, 1928} \\
\hline 48 & Stiphrolamyra rubicunda Oldroyd, 1947 & Saghaei et al., 2008, 2009, 2010 \\
\hline \multicolumn{3}{|c|}{ Subfamily Laphystiinae Hendel, 1936} \\
\hline \multicolumn{3}{|c|}{ Genus Laphystia Loew, 1847} \\
\hline 49 & Laphystia erberi (Schiner, 1865) & Saghaei et al., 2008, 2009, 2010 \\
\hline 50 & Laphystia latiuscula Loew, 1871 & Abbassian-Lintzen, 1964 \\
\hline 51 & Laphystia sabulicola Loew, 1847 & Ghahari et al., 2007b \\
\hline \multicolumn{3}{|c|}{ Subfamily Leptogastrinae Schiner, 1862} \\
\hline \multicolumn{3}{|c|}{ Genus Leptogaster Meigen, 1803} \\
\hline 52 & Leptogaster cylindrica (De Geer, 1776) & Saghaei et al., 2008, 2009, 2010 \\
\hline 53 & Leptogaster nartshukae Lehr, 1961 & Present study \\
\hline
\end{tabular}

Subfamily Ommatiinae Hardy, 1927

Genus Ommatius Wiedemann, 1821

54 Ommatius tenellus Wulp, 1889

Present study

Subfamily Stenopogoninae Hull, 1962

Tribe Dioctriini Enderrleini, 1936

Genus Dioctria Meigen, 1803

55 Dioctria striata Theodor, 1980

Lehr et al., 2007

Genus Molobratia Hull, 1958

56 Molobratia egregia (Loew, 1869)

Lehr et al., 2007

57 Molobratia teutonus (Linnaeus, 1767) Tomasovic and Saghaei, 2009

Tribe Stichopogonini Hardy, 1930

Genus Stichopogon Loew, 1847

58 Stichopogon chrysostoma Schiner, 1867 Present study

Tribe Stenopogonini Hardy, 1930

Genus Amphisbetetus Hermann, 1906

59 Amphisbetetus favillaceus Saghaei et al., 2008, 2009

(Loew in Rosenhauer, 1856)

Genus Ancylorhynchus Berthold, 1827

60 Ancylorhynchus argyrogaster (Seguy, 1932)

Tomasovic and Saghaei, 2009 
Table 1 (cont.)

\begin{tabular}{lll}
\hline No. & Subfamily, Tribe, Genus and Species & References \\
\hline
\end{tabular}

Genus Anisopogon Loew, 1874

61 Anisopogon hermanni (Engel, 1930)

Lehr et al., 2007

Genus Crobilocerus Loew, 1847

62 Crobilocerus megilliformis (Loew, 1847)

Abbassian-Lintzen, 1964

63 C. spinosus persianus Geller-Grimm and Hradský, 1999

Saghaei et al., 2008, 2009, 2010;

Tomasovic and Saghaei, 2009

\section{Genus Cyrtopogon Loew, 1847}

64 Cyrtopogon gorodkovi Lehr, 1966

Sakenin et al., 2008

Genus Galactopogon Engel, 1929

65 Galactopogon hispidus Engel, 1929

Abbassian-Lintzen, 1964;

Saghaei et al., 2008, 2009, 2010; Present study

Genus Habropogon Loew, 1847

66 Habropogon decipiens Theodor, 1980

Saghaei et al., 2008, 2009

Genus Heteropogon Loew, 1847

67 Heteropogon nubilus (Wiedeman in Meigen,

Lehr et al., 2007 1820)

68 Heteropogon pyrinus Hermann, 1906

Saghaei et al., 2008, 2009

Genus Iranopogon Timon-David, 1955

69 Iranopogon brandti Timon-David, 1955

Timon-David, 1955

\section{Genus Pycnopogon Loew, 1847}

$70 \quad$ Pycnopogon mixtus (Loew, 1847)

Saghaei et al., 2008, 2009, 2010

\section{Genus Sisyrnodytes Loew, 1856}

71 Sisyrnodytes nilicola (Rondani, 1850)

Sakenin et al., 2008

Genus Stenopogon Loew, 1847

72 Stenopogon callosus (Pallas in Wiedemann, 1818)

73 Stenopogon elongatus (Meigen, 1804)

74 Stenopogon heteroneurus (Macquart, 1838)

Lehr et al., 2007

75 Stenopogon junceus (Wiedemann in Meigen, 1820)

76 Stenopogon laevigatus (Loew, 1851)

77 Stenopogon sciron (Loew, 1873)

Saghaei et al., 2010

Abbassian-Lintzen, 1964; Saghaei et al., 2010

Abbassian-Lintzen, 1964; Saghaei et al., 2008, 2009, 2010; Tomasovic and Saghaei, 2009

Abbassian-Lintzen, 1964

Saghaei et al., 2008, 2009, 2010;

Tomasovic and Saghaei, 2009;

Present study

78 Stenopogon shirazi Tomasovic and Saghaei,

Tomasovic and Saghaei, 2009

2009

79 Stenopogon strataegus Gerstaecker, 1862

Lehr et al., 2007 


\section{Discussion}

In the course of the present study a total of 12 species belonging to 12 genera and 6 subfamilies were collected and identified. Of these, one species (Tolmerus paganus Becker, 1923) is considered as new record for Iranian Asilid fauna. As a result of this study one subfamily (Ommatiinae Hardy, 1927), 2 genera (Ommatius Wiedemann, 1821 and Stichopogon Loew, 1847), and four species were added, bringing the totals to 79 asilid species in 43 genera and 8 subfamilies in Fars province (Table 1).

The greatest number of species was that of the subfamily Asilinae, with 31 species and 16 genera. The proportions in other subfamilies were as followed: Stenopogoninae (25 species and 15 genera), Apocleinae (6 species and 3 genera), Dasypogoninae (6 species and 2 genera), Laphrinae ( 5 species and 2 genera), Laphystiinae ( 3 species and 1 genus), Leptogastrinae ( 2 species and 1 genus) and Ommatiinae ( 1 species and 1 genus).

Currently, the subfamily Atomosiinae, are not present in Fars province. In other parts of Iran, from this subfamily, two species Amathomyia persiana Hermann, 1912 and Loewinella virescens (Loew, 1871) have been recorded from Baluchestan (Becker and Stein, 1913) and Khuzestan (Oldroyd 1958) provinces respectively before.

One subspecies: Aneomochtherus ochriventris persicus Tomasovic and Saghaei, 2009 and 6 species: Erax grootaerti Tomasovic, 2002; Paramochtherus persicus Tomasovic and Saghaei, 2009; Andrenosoma farsicola Tomasovic and Saghaei, 2009; Ancylorhynchus argyrogaster (Seguy, 1932); Iranopogon brandti Timon-David, 1955; Stenopogon shirazi Tomasovic and Saghaei, 2009 have been described from Fars province and they haven't been cited from other parts of Iran and the world from today.

Although the fauna of Asilidae in Fars province were studied (Saghaei et al., 2008, 2009, 2010; Tomasovic and Saghaei 2009), but co-evolution and biology of almost species and their prey are unknown. The species of Asilidae are important in biological control of other arthropods (Lavigne and Dennis, 1994; Londt, 1994, 2006; Dennis and Lavigne, 2007), so further study of their occurrence, distribution, biology and behavior in Iran should be encouraged.

\section{Acknowledgements}

The research was supported by Islamic Azad University, Marvdasht Branch, Marvdasht, Iran. The author is indebted to Dr. Guy Tomasovic (Belgium) for his invaluable help with the project.

\section{Literature}

Abbassian-Lintzen, R. (1964): Asilidae (Diptera) of Iran. I. Robber flies belonging to the subfamilies Laphriinae and Dasypogoninae (with description of new species). Ann. Mag. Nat. Hist. 13, 417-435.

Becker, T. and Stein, P. (1913): Persische Dipteren von den Expeditionen des Herrn N. A. Zarudny 1898 und 1901. Annulaire du Musee Zoologique de 1'Academia Imperiale des Sciences de St. Petersbourg 17, 503-654. 
Bosák, J. and Hradský, M. (2001): Some remarks on the distribution of robber flies (Diptera: Asilidae) in Turkey. J. Entomol. Res. Soc. 3, 1-28.

Dennis, D. S. and Lavigne, R. J. (2007): Hymenoptera as prey of robber flies (Diptera: Asilidae) with new prey records. J. Entomol. Res. Soc. 9, 23-42.

Engel, E. O. (1930): 24. Asilidae. In: E. Lindner (ed.): Die Fliegen der Palaarktischen Region Band IV (2) [9 parts: 1925-1930, complete book: 1938]. Stuttgart: Schweizerbart, 491 p.

Geller-Grimm, F., Dikow, T. and Lavinge, R. J. (2015): Robber Flies (Asilidae) Database, Species. http://www. geller-grimm.de/catalog/species.htm, accessed: January 12, 2015.

Ghahari, H., Hayat, R., Ostovan, H. and Lavigne R. (2007a): Robber flies (Diptera: Asilidae) of Iranian rice fields and surrounding grasslands. Linzer Biol. Beitr. 39/2, 919-928.

Ghahari, H., Lehr, P. A., Lavigne, R. J., Hayat, R. and Ostovan, H. (2007b): New records of robber flies (Diptera, Asilidae) for the Iranian fauna with their prey records. Far East. Entomol. 179, 1-9.

Hayat, R., Ghahari H., Lavigne, R. and Ostovan, H. (2008): Iranian Asilidae (Insecta: Diptera). Turk. J. Zool. 32, 175-195.

Lavigne, R. J. and Dennis, S. (1994): New records of Coleoptera as prey for Asilidae in Wyoming and Colorado. Coleopts Bull. 48, 131-139.

Lehr, P. A. (1988): Asilidae. In: A. Soos and L. Papp (eds): Catalogue of Palearctic Diptera. Elsevier Science Publishing Co. Inc., Amsterdam, pp. 197-326.

Lehr, P. A., Ghahari, H. and Ostovan, H. (2007): A contribution to the robber flies of Subfamilies Stenopogoninae and Asilidae (Diptera: Asilidae) from Iran. Far East. Entomol. 173, 1-14.

Londt, J. G. H. (1993): Afrotropical robber fly (Diptera: Asilidae) predation of honey bees, Apis mellifera Linnaeus (Hymenoptera: Apidae). Afr. Entomol. 1, 167-173.

Londt, J. G. H. (1994): Afrotropical Asilidae (Diptera) 26. Ethological observations, and a possible ecological classification based on habitats. Ann. Natal Mus. 35, 97-122.

Londt, J. G. H. (1995): Afrotropical Asilidae (Diptera) 27. Predation of Asilidae by Asilidae. Ann. Natal Mus. $36,161-167$.

Londt, J. G. H. (1996): Afrotropical Asilidae (Diptera) 28. A review of the genus Oxynoton Janssens, 1951 and its transfer from the subfamily Trigonomiminae to the Stenopogoninae. Ann. Natal Mus. 37, 173-181.

Londt, J. G. H. (2006): Predation by Afrotropical Asilidae (Diptera): an analysis of 2000 prey records. Afr. Entomol. 14, 317-328.

Oldroyd, H. (1958): Some Asilidae from Iran. Stutt. Beitr. Naturkd. 9, 1-10.

Saghaei, N., Ostovan, H. and Shojaei, M. (2008): A faunistic study of robber flies (Asilidae) in Fars province. Proc. of 18th Iranian Plant Protection Congress, University of Bu-Ali Sina Hamedan, 88 p.

Saghaei, N., Ostovan, H., Shojaei, M. and Hayat, R. (2009): Introduction to the Asilidae fauna (Insecta: Diptera) of Fars province, Iran. Turk. J. Zool. 33, 187-200.

Saghaei, N., Hayat, R., Ostovan, H. and Fallahzadeh, M. (2010): Prey of some robber flies (Diptera: Asilidae) in Fars province, Iran. J. Entomol. Res. Soc. 12, 17-26.

Sakenin, H., Eslami, B., Samin, N., Imani, S., Shirdel, F. and Havaskary, M. (2008): A contribution to the most important trees and shrubs as the hosts of wood-boring beetles in different regions of Iran and identification of many natural enemies. J. Plant Ecos. 16, 27-46.

Sakenin, H., Samin, N., Ghahari, H., Imani, S., Rastegar, J. and Jabbari, A. (2010): A contribution to the knowledge of robber flies (Diptera: Asilidae) from Semnan province, Iran. Linzer biol. Beitr 42, 833-841.

Samin, N., Sakenin, H. and Imani, S. (2011a): A contribution to the knowledge of robber flies (Diptera: Asilidae) from some regions of Iran. Calodema 159, 1-5.

Samin N., Zhou, H., Imani, S. and Rastegar, J. (2011b): A contribution to the knowledge of Iranian Staphylinidae (Coleoptera: Staphylinoidea). Arch. Biol. Sci. 63, 1235-1243.

Theodor, O. (1980): Diptera: Asilidae. Fauna Palaestina, Insecta II. Israel Academy of Sciences and Humanities, Jerusalem, $448 \mathrm{p}$.

Timon-David, J. (1955): Iranopogon brandti, n. gen., n. sp., Asilidae xerophile d'iran (Dipt.). Bull. Soc. Entomol. Fr. 60, 102-104. 
Tomasovic, G. (1999): Notes sur les Asilidae palearctiques (Diptera Brachycera) (10 et 11). Description et repartition geographique de 2 especes nouvelles de Dasypogon du groupe diadema (Fabricius, 1781). Bull. Ann. Soc. R. Belge. Entomol. 135, 216-221.

Tomasovic, G. (2002): Etude sur materiaux typiques du complexe genital male de spet espece du genre Erax scopoli, 1763 (Diptera: Asilidae) avec la description de trois especes nouvelles. Entomologie faunistique-Faunistic Entomology 46, 27-37.

Tomasovic, G. and Saghaei, N. (2009): Contribution to the knowledge of the Asilidae (Diptera: Brachycera) from Fars province (Iran). Entomologie faunistique-Faunistic Entomology 62, 45-56.

Tsacas, L. (1968): Revision des especes du genere Neomochtherus Osten-Sacken (Dipteres: Asilidae). I. Region palearctique. [Revision of the species of the genus Neomochtherus Osten-Sacken (Diptera: Asilidae). I. Palearctic region]. Mém. Mus. Natl Hist. Nat. (N.S.), Ser. A. Zoology 47, 129-328.

Wood, G. C. (1981): Asilidae. In: J. P. McAlpine et al. (eds): Manuel of Nearctic Diptera, Vol. 1; Ottawa: Research Branch, Agriculture Canada, Monograph 27, pp. 549-573. 